[0212-7199 (2007) 24: 3; pp 132-134] ANALES DE MEDICINA INTERNA Copyright (C) 2007 ARAN EDICIONES, S.L.

An. MEd. InTERna (Madrid) Vol. 24, N. $^{\circ}$ 3, pp. $132-134,2007$

\title{
Degeneración cerebelosa paraneoplásica como presentación de una recidiva de carcinoma de mama
}

\author{
I. GÁZQUEZ SISTERÉ, S. CARO OROZCO, D. MÁRQUEZ MEDINA', J. CHORDÁ \\ RIBELLES, A. QUÍLEZ MARTÍNEZ ${ }^{2}$
}

Servicios de Medicina Interna, ${ }^{\prime}$ Oncología Médica $y^{2}$ Neurología. Hospital Universitario Arnau de Vilanova. Lleida

PARANEOPLASTIC CEREBELLAR DEGENERATION AS A PRESENTATION OF BREAST CANCER RECURRENCE.

\begin{abstract}
RESUMEN
La degeneración cerebelosa paraneoplasica (DCP) es un síndrome paraneoplásico poco frecuente que se caracteriza por disfunción cerebelosa global. La DCP a menudo precede meses o años a una neoplasia potencialmente curable, con menos frecuencia ocurre en pacientes con neoplasia conocida o indica recurrencia. La presencia de anticuerpos específicos en suero ayuda a guiar la identificación de una neoplasia oculta. Comunicamos el caso de una paciente de 74 años que presentó disfunción cerebelosa. Había sido diagnosticada de carcinoma ductal de mama hacia 5 años, permaneciendo asintomática hasta entonces. Con la pruebas de imagen convencionales no fue posible detectar recidiva tumoral, el diagnóstico se realizó con la ayuda de la tomografía con emisión de positrones que detectó adenopatías abdominales. Revisamos brevemente las características clínicas de este síndrome, remarcando la importancia de un rápido reconocimiento del síndrome, que en muchas ocasiones permite una rápida detección y tratamiento de la enfermedad primaria.
\end{abstract}

PALABRAS CLAVE: Degeneración cerebelosa paraneoplásica. Cáncer de mama. Anticuerpos antineuronales.

\begin{abstract}
Paraneoplastic cerebellar degeneration (PCD) is a rare condition which is characterised by global cerebellar dysfunction. Patients may present with these syndromes months or years before the diagnosis of underlying malignancy is established. Less often, PCD occurs in patients with a known malignancy or heralds the onset of a recurrence. The presence of specific antibodies in serum simples helps to guide identification the occult malignancy. We report here the case of a PCD in 74-year-old lady underwent a left mastectomy for breast cancer 5 years ago. She remained well until now. The diagnosis of the primary tumor, that is clinically undetectable with conventional imaging processes, is preformed with the aid of positron mission tomography (PET) to detect the presence of abdominal lymph node metastases. We briefly review the clinical and laboratory features of this syndrome, and emphasize the importance of its prompt recognition, which many times makes possible the early detection and treatment of the primary disorder.
\end{abstract}

KEY WORDS: Paraneoplastic cerebellar degeneration. Breast cancer. Antineuronal antibodies.

Gázquez, Sisteré I, Caro Orozco S, Márquez Medina D, Chordá Ribelles J, Quílez, Martínez. A. Degeneración cerebelosa paraneoplásica como presentación de una recidiva de carcinoma de mama. An Med Interna (Madrid) 2007; 24: 132-134.

\section{INTRODUCCIÓN}

La degeneración cerebelosa paraneoplásica (DCP) es una entidad rara incluida dentro de los llamados síndromes neurológicos paraneoplásicos. Clínicamente se caracteriza por disfunción cerebelosa subaguda y progresiva. El carcinoma pulmonar de células pequeñas es la causa neoplásica más frecuente de DCP. Otros tumores asociados son el carcinoma de mama, de ovario y la enfermedad de Hodgkin $(1,2)$. En pacientes mayores de 50 años la degeneración cerebelosa aguda o subaguda es paraneoplásica en el $50 \%$ de los casos y se asocia a una neoplasia oculta potencial- mente curable (2). Con menos frecuencia la DCP ocurre en pacientes con neoplasia conocida o alerta sobre una recurrencia (1-3). Se han encontrado en suero y líquido cefalorraquídeo (LCR) de pacientes con DCP varios tipos de anticuerpos antineuronales incluyendo anti-Yo, anti-Ri y anti-Tr (4,5). La presencia de un anticuerpo determinado puede orientar sobre el origen de neoplasia (5), así la presencia de anticuerpos anti-Yo se relaciona con carcinoma de mama y ginecológico (6). En nuestro caso la forma de presentación de la recidiva de carcinoma de mama con un cuadro paraneoplásico contribuyó al diagnóstico y tratamiento antitumoral precoz.

Trabajo aceptado: 25 de octubre de 2006 


\section{CASO APORTADO}

Una mujer de 74 años ingreso en nuestro centro por presentar desde hacia una semana sensación de vértigo rotatorio con nauseas y vómitos, inestabilidad de la marcha, diplopía binocular horizontal y alteraciones del habla. Entre los antecedentes personales destacaba alergia a contraste iodado y carcinoma de mama ductal infiltrante con receptores a estrógenos y progesterona positivos y ganglios negativos tres años antes. Realizó tratamiento quirúrgico y radioterapia de la mama residual. Actualmente estaba en remisión y seguía tratamiento con anastrozol. La exploración física puso de manifiesto un habla escandida, nistagmo con componente rápido rotatorio en todas las direcciones de la mirada, paresia de IV par craneal derecho, ataxia truncal invalidante con imposibilidad para la marcha y dismetria en las cuatro extremidades. Las funciones corticales superiores estaban conservadas. En los análisis generales se evidenció: un hemograma bioquímica general y hemostasia normales. Los marcadores tumorales habituales (CA 125, CA 15-3, CA19-9, alfafetoproteína y antígeno carcinoembrionario) eran normales. Se realizó un estudio de neuroimagen con tomografía axial computarizada (TC) y resonancia magnética (RM) cerebrales que fueron normal. Se sospechó DCP y se determinaron los anticuerpos anti-Yo que fueron positivos a títulos altos en suero y en LCR. Mientras se realizaba el estudio de neoplasia oculta se inició tratamiento con inmunoglobulinas, ciclofosfamida y metilprednisolona sin mejoría clínica evidente. En la TC toracoabdominal, mamografía, RM de mama y gammagrafía ósea no se evidenció patología tumoral. La tomografía con emisión de positrones (PET) mostró dos lesiones nodulares de $15 \mathrm{~mm}$ localizadas detrás de la cabeza pancreática compatibles con adenopatías mesentéricas de etiología maligna. No se observaron otros focos sospechosos de malignidad. Se realizó laparotomía y biopsia. El estudio anatomopatológico mostraba la presencia de metástasis de adenocarcinoma mal diferenciado. Se consideró el diagnostico de metástasis de carcinoma de mama y el Servicio de Oncología Médica inició tratamiento con epirubicina y ciclofosfamida. La evolución clínica de la paciente fue hacia la estabilización sin mejoría de signos y síntomas, la paciente no era capaz de realizar sola las actividades de la vida diaria y necesitaba silla de ruedas para los desplazamientos

\section{DISCUSIÓN}

El DCP es un síndrome paraneoplásico del sistema nervioso raro pero bien caracterizado, con una incidencia de menos del $1 \%$ en los pacientes con cáncer (7). Clínicamente, los pacientes presentan ataxia, disartria, temblor de intención, nistagmo, vértigo y diplopía (4). Se han descrito en el $50 \%$ de los pacientes síntomas y signos de afectación neurológica extracerebelosa (8). Las funciones cognitivas están respetadas. El cuadro de DCP se desarrolla en el curso de semanas a meses y, en más de la mitad de los casos, precede meses o años a los síntomas del tumor (1), con menos frecuencia alerta sobre la recurrencia del cáncer o se presentar en una neoplasia conocida (9). En nuestro caso, se trataba de una paciente con carcinoma de mama y libre de enfermedad con el seguimiento habitual, en la que la DCP alertó sobre la posible recidiva de la enfermedad y además solo el PET mostró la existencia de metástasis.

El diagnóstico de DCP depende de la clínica y de la existencia de anticuerpos antineuronales $(10,11)$. Aunque estos no son necesarios para el diagnóstico (11). El proceso se considera mediado inmunologicamente. Evidencias como el hallazgo de anticuerpos dirigidos contra proteínas especificas compartidas por las células de Purkinje y tumorales, soportan la etiología autoinmune de este proceso (10). Se ha descrito la pre- sencia de anticuerpos anti-Hu en pacientes con carcinoma pulmonar de células pequeñas (12), anticuerpos anti-Ri y anti-Yo en el cáncer de mama y anticuerpos anti-Tr en la enfermedad de Hodgkin $(5,13)$. Los anticuerpos anti-Yo se asocian a cáncer mama o ginecológico en más del $95 \%$ de los casos $(6,11)$. Así los ac anti-Yo son un excelente marcador diagnostico en la DCP de cáncer de mama o ginecológico subyacente $(11,14)$. Los anticuerpos se encuentran en suero y en LCR y pueden ser medidos cuantitativamente, aunque la determinación de títulos de anticuerpos en suero o en LCR no es necesaria para el diagnostico (6). El análisis western blot de anticuerpos anti-Yo no tiene falsos positivos conocidos, y no se detectan en individuos normales (6). Aunque algunas publicaciones muestran que aproximadamente el $20 \%$ de los pacientes con cáncer de ovario sin síndromes neurológicos también expresa antígenos Yo en el tumor (15). No se conoce la razón por la que estos pacientes no desarrollan DCP.

La histología del DCP tiene como signo fundamental la pérdida difusa de células de Purkinje asociado en ocasiones a un infiltrado inflamatorio en las áreas afectadas del sistema nervioso $(2,16)$.

Realizar estudios controlados avalando los diversos esquemas terapéuticos para el DCP se encuentra con varias dificultades como la rareza de estos síndromes que no permite establecer la ocurrencia de estabilización o remisión espontánea. En la actualidad no existe un tratamiento establecido que mejore significativamente los síntomas de DCP. Excepto en casos individuales los intentos de tratarlos síntomas neurológicos con inmunoglobulinas, quimioterapia o imnunosupresión no producen los efectos deseados sobre los síntomas (1719). También se ha examinado el efecto del tratamiento combinado empleando immunoglubulinas, ciclofosfamida y metilprednisolona en pacientes con DCP y anticuerpos antiYo (20). Los pacientes toleraron bien el tratamiento pero este fracasó en producir el efecto clínico deseado de forma significativa, solo en un caso con una afectación menos severa se observo una estabilización transitoria de los síntomas (20). La mala respuesta al tratamiento de los síndromes neurológicos paraneoplásicos se ha atribuido a un inicio del tratamiento cuando la pérdida neuronal era ya masiva e irreversible (20). En cuanto al tratamiento antineoplásico (cirugía o quimioterapia) no todos los trabajos coinciden en su efecto del sobre los síntomas neurológicos. En algunas publicaciones se muestra que el tratamiento antineoplásico (cirugía o quimioterapia) es el único que se asocia de forma significativa con estabilización o mejoría de los síntomas neurológicos $(21,22)$, mientras que en otras la diferencia no es significativa $(11,13)$. No siendo posible determinar si la mejoría es debida a la disminución de antígenos por la exéresis del tumor o es consecuencia del efecto inmunosupresor del tratamiento antineoplásico. Además, en muchas ocasiones el estado general de pacientes en tratamiento con quimioterapia se deteriora demasiado deprisa para valorar los efectos positivos de tratamiento en el DCP. En estudios previos se ha observado que el único factor independiente para la supervivencia de pacientes con DCP y anticuerpos anti-Yo es el tipo de tumor asociado siendo la media de supervivencia de 100 meses en los pacientes con cáncer de mama (23). Siendo la progresión del tumor la causa de la muerte en el 29-52\% de los casos según las series $(13,22,23)$. En nuestra paciente ni el tratamiento inmunosupresor precoz iniciado antes del diagnostico definitivo de la neoplasia ni el tratamiento del tumor (exéresis de las adenopatías y quimiote- 
rapia) lograron una mejoría de los síntomas neurológicos. Aunque la presencia de la DCP sí permitió un diagnóstico precoz de la neoplasia y posiblemente una mejor supervivencia aunque con persistencia de los síntomas neurológicos.

En resumen: El diagnostico correcto de la DCP depende de un alto grado de sospecha por parte del clínico. La evidencia de anticuerpos específicos en suero puede ser útil en la búsqueda del tumor primario, ya que anticuerpos específi- cos se asocian con frecuencia a un tumor determinado (5). Sin embargo, como en el caso que presentamos, los procedimientos diagnósticos habituales pueden no ser suficientes para llegar al diagnostico y solo el PET permite realizar el diagnóstico de neoplasia oculta (24). Por tanto, su importancia reside en la oportunidad de identificar y tratar precozmente el tumor que en muchas ocasiones escapa a los controles periódicos.

\section{Bibliografía}

1. Posner JB. Paraneoplastic syndromes. In: Neurologic complications of cancer. Philadelphia: FA Davis; 1995. p. 353-85.

2. Henson RA, Urich H, eds. Cancer and the Nervous system: The Neurological Manifestations of Systemic Malignant Disease. Oxford, England: Black-well Scientific Publications; 1982. p. 346-367.

3. Greenlee JE, Brashear HR, Jaeckle KA, Geleris A, Jordan K. Pursuing an occult carcinoma in a patient with subacute cerebellar degeneration and anticerebellar degeneration and anticerebellar antibodies: need for vigorous follow-up. West J Med 1992; 156: 199-202.

4. Anderson NE, Rosenbulm MK, Posner JB. Paraneoplastic cerebellar degeneration: clinical-immunological correlations. Ann Neurol 1988; 24: 559-567.

5. Dalmau JO, Posner JB. Paraneoplastic syndromes. Arch Neurol 1999; 56: 405-408.

6. Peterson K, Rosenblum MK, Kotanides H, Posner JB. Paraneoplastic cerebellar degeneration, I: A clinical analysis of 55 anti-Yo antibodypositive patients. Neurology 1992; 42: 1931-1937

7. Moll JWB, Hooijkaas H, van Goorbergh BCM, Roos LGE, HenzenLogmans SC, Vecht CJ (1996) Systemic and antineuronal auto-antibodies in patients with paraneoplastic neurological disease. J Neurol 243: 51-56.

8. Posner JB. Paraneoplastic cerebellar degeneration. Can J Neurol Sci 1993; 20 (Supl. 3): S117-S122.

9. Hammack JE, Kimmel DW, O’Neill BP, Lennon VA. Paraneoplastic cerebellar degeneration: a clinical comparison of patients with and without Purkinje cell cytoplasmic antibodies. Mayo Clin Proc 1990; 65: 1423-1431.

10. Moll JWB, Antoine JC, Brashear HR, Delattre J, Drlicek M, Dropcho EJ, et al. Guidelines on the detection of paraneoplastic anti-neuronal specific antibodies: report from Workshop to the Fourth meeting of the International Society of Neuro-immunology on paraneoplastic neurological disease, held October 22-23, 1994, in Rotterdam, The Netherlands. Neurology 1995; 45: 1937-1941.

11 Rojas-Marcos I, Rousseau A, Keime-Guibert F, René R, CartalatCarel S, Delattre JY, Graus F. Spectrum of paraneoplastic neurologic disorders in women with breast and gynaecologic cancer. Medicine 2003; 82: 216-223.

12. Graus F, Keime-Guibert F, Reñe R, Benyahia B, Ribalta T, Ascaso C, et al. Anti-Hu-associated paraneoplastic encephalomyelitis: analysis of 200 patients. Brain 2001; 124: 1138-1148.

13. Shams'ili S, Grefkens, de Leeuw B, van den Bent M, Hooijkaas H, van der Holt B, et al. Paraneoplastic cerebellar degeneration associa- ted with antineuronal antibodies: analysis of 50 patients. Brain 2003; 126: $1409-1418$.

14. Waterhouse DM, Natale RB, Gody RL. Breast cancer and paraneoplastic cerebellar degeneration. Cancer 1991; 68: 1835-1841.

15. Liu S, Mezrich J, Berk J, Dalmau J, Posner JB. Expression of Purkinje cell antigens in ovarian tumor and the presence of anti-Purkinje cell antibodies in the serum of patients without paraneoplastic cerebellar degeneration. Neurology 1994; 45 (Supl. 4): A288-A229. Abstract.

16. Furneaux HM, Rosenblum MK, Dalmau J, Wong E, Woodruff P, Graus F, Posner JB. Selective expression of Purkinje cell antigens in tumor tissue from patients with paraneoplastic cerebellar degeneration. N Engl J Med 1990; 322: 1844-1851.

17. Phuphanich S, Brock C. Neurologic improvement after high-dose intravenous immunoglobulin therapy in patients with paraneoplastic cerebellar degeneration associated with anti-Purkinje cell antibody. J Neurooncol 2006; 14 June, online.

18. Widdess-Walsh P, Tavee JO, Schuele S, Stevens GH. Response to intravenous immunoglobulin in anti-Yo associated paraneoplastic cerebellar degeneration: case report and review of the literature. J Neurooncol 2003; 63: 187-190.

19. Blaes F, Strittmatter M, Merkelbach S, Jost V, Klotz M, Schimrigk K, Hamann GF. Intravenous immunoglobulins in the therapy of paraneoplastic neurological disorders. J Neurol 1999; 246: 299-303.

20. Keime-Guibert F, Graus F, Fleury A, René R, Honnorat J, Broet P, Delattre JY. Treatment of paraneoplastic neurological syndromes with antineuronal antibodies (Anti-Hu, Anti-Yo) with a combination of immunoglobulins, cyclophosphamide, and methylprednisolone. J Neurol Neurosurg Psychiatry 2000; 68: 479-48.

21. Faris M, Abraham J, Barret-Lee P. A patient with breast cancer and paraneoplastic cerebellar syndrome associated with anti-Purkinje cell antibodies: response to CMF chemotherapy. Clin Oncol (R Coll Radiol) 1998; 10: 202-203.

22. Candler PM, Hart PE, Barnett M, Weil R, Rees JH. A follow up study of patients with paraneoplastic neurological disease in the United Kingdom. J Neurol Neurosurg Psychiatry 2004; 75: 14111415.

23. Rojas-Marcos I, Graus F, Keime-Guibert F, Reñe R, Delattre JY, Ramon JM, et al. Long-term clinical outcome of paraneoplastic degeneration and anti-Yo antibodies. Neurology 2000; 55: 713-715.

24. Berner U, Menzel C, Rinne D, Kriener S, Hamscho N, Döbert N, et al Paraneoplastic syndromes: detection of malignant tumors using [18F]FDG-PET. Q J Nucl Med 2003; 47: 85-89. 